Revue d'histoire de l'Amérique française

REVUE D.HISTOIRE DE L'AMÉRIQUE FRANÇAISE

\title{
Les activités forestières dans deux communautés agricoles du Nouvel-Ontario,1900-1920
}

\section{Guy Gaudreau}

Volume 54, numéro 4, printemps 2001

URI : https://id.erudit.org/iderudit/005439ar

DOI : https://doi.org/10.7202/005439ar

Aller au sommaire du numéro

Éditeur(s)

Institut d'histoire de l'Amérique française

ISSN

0035-2357 (imprimé)

1492-1383 (numérique)

Découvrir la revue

Citer cet article

Gaudreau, G. (2001). Les activités forestières dans deux communautés agricoles du Nouvel-Ontario,1900-1920. Revue d'histoire de l'Amérique française, 54(4), 501-529. https://doi.org/10.7202/005439ar
Résumé de l'article

L'examen des activités forestières à l'échelle locale au cours des deux premières décennies du xxe siècle permet à l'auteur d'ajouter une nouvelle perspective à l'étude de ces activités en milieu agricole. Les terres et les forêts de deux communautés canadiennes-françaises du Nouvel-Ontario, Blezard et Hanmer, ont été découpées sur une base cantonale et ont fait l'objet de nombreuses évaluations consignées dans deux sources gouvernementales, soit le registre des concessions forestières - plus connu des chercheurs - et le registre de coupes du district de Sudbury, document majeur, méconnu et souvent disparu dans les autres régions. Les données recueillies permettent de relativiser la place des activités de coupe sur les lots agricoles et d'esquisser l'hypothèse de trois étapes dans le déroulement de l'abattage de la matière ligneuse. 


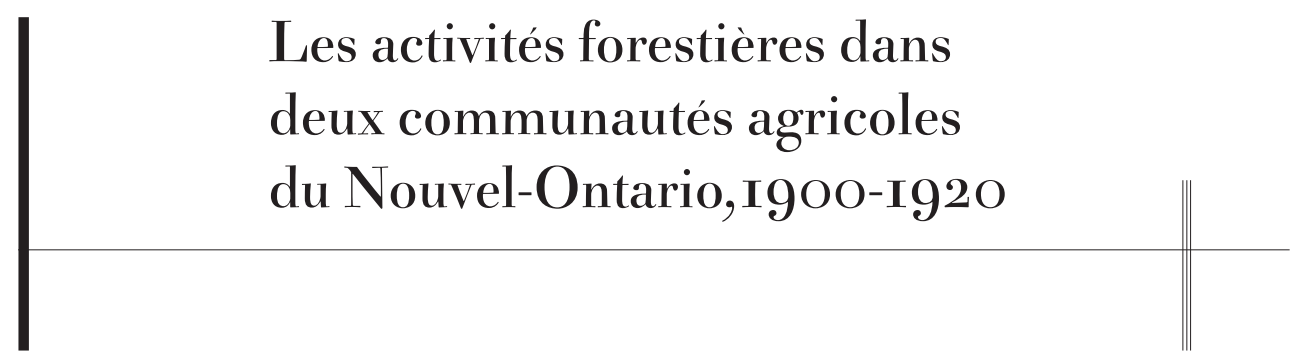

GUY GAUDREAU

Département d'histoire

Université Laurentienne

RÉSUMÉ - L'examen des activités forestières à l'échelle locale au cours des deux premières décennies du $\mathrm{XX}^{\mathrm{e}}$ siècle permet à l'auteur d'ajouter une nouvelle perspective à l'étude de ces activités en milieu agricole. Les terres et les forêts de deux communautés canadiennes-françaises du Nouvel-Ontario, Blezard et Hanmer, ont été découpées sur une base cantonale et ont fait l'objet de nombreuses évaluations consignées dans deux sources gouvernementales, soit le registre des concessions forestières — plus connu des chercheurs - et le registre de coupes du district de Sudbury, document majeur, méconnu et souvent disparu dans les autres régions. Les données recueillies permettent de relativiser la place des activités de coupe sur les lots agricoles et d'esquisser l'hypothèse de trois étapes dans le déroulement de l'abattage de la matière ligneuse.

ABSTRACT • In examining forestry activities during the first two decades of the twentieth century at a local level - specifically, two French Canadian communities in the Sudbury area of "New Ontario" - the author provides new insights into the history of the industry in an agricultural setting. Drawing principally upon two provincial governmental sources often not extant or available to researchers, the Ontario Department of Lands and Forests (originally Crown Lands) Registry of Timber Licences and the Timber Agent's Book for the Sudbury District, the paper analyses harvesting operations in Blezard and Hanmer, and offers an hypothesis of a three-step process to explain the evolving forestry-agriculture relationship. 
$\mathrm{H}^{2}$ ANMER ET Blezard sont deux communautés agricoles du NouvelOntario qui, en dépit de leur nom, ont une origine canadiennefrançaise ${ }^{1}$. Fondées au tournant $\mathrm{du} \mathrm{xx}^{\mathrm{e}}$ siècle, elles font alors partie de ces paroisses de colonisation que le Canada français égrène de la vallée laurentienne jusqu'aux Rocheuses ${ }^{2}$. Situées seulement à une vingtaine de kilomètres au nord de Sudbury, elles connaissent néanmoins un développement qui, au cours des premières décennies de leur existence, reste peu tributaire de la hausse des activités minières régionales ${ }^{3}$.

De vocation surtout agricole, les terres enserrant ces deux communautés voisines s'étalent sur six cantons totalisant plus de 500 kilomètres carrés. Ces six cantons, identifiés sur la carte 1 , font partie d'une région agricole plus vaste appelée communément «La Vallée». Si ces cantons ont été réunis pour fin d'analyse, c'est qu'ils ont constitué pendant 30 ans (1969-1999), une municipalité indépendante, Vallée-Est ${ }^{4}$.

Les forêts de ces cantons ont fait l'objet d'une coupe intensive à partir de la fin du $\mathrm{XIX}^{\mathrm{e}}$ siècle. Dès 1920, l'essentiel des espèces commerciales était liquidé. Il est possible de reconstituer avec précision ces activités, car elles sont consignées dans des registres de coupe conservés dans les archives publiques et qui feraient l'envie de bien des chercheurs ${ }^{5}$.

1. Pour une vue d'ensemble de l'histoire du Nord ontarien, voir Gaétan Gervais, Matt Bray et Ernie Epp, dir., Un vaste et merveilleux pays (Toronto, Ministère des Affaires du Nord de l'Ontario, 1984).

2. Deux ouvrages récents sur la francophonie canadienne abordent l'histoire de ces différentes communautés; voir Joseph Yvon Thériault, dir., Francophonies minoritaires au Canada. L'état des lieux (Moncton, Éditions d'Acadie, 1999), voir notamment l'article de Gaétan Gervais, "L'Histoire de l'Ontario français», 145-161; Gratien Allaire, La francophonie canadienne. Portraits (Québec/Sudbury, AFI-CIDEF/Prise de parole, 1999); le chapitre IV traite exclusivement de l'Ontario français.

3. Ce n'est qu'après la Deuxième Guerre mondiale que la population des communautés agricoles de la région quittera massivement le travail de la terre pour le travail minier; voir à ce sujet, Huguette Parent, Le Township de Hanmer, 1904-1969 (Sudbury, Société historique du NouvelOntario, 1979), 30; Donald Dennie, «La propriété foncière à Chelmsford et dans le canton de Balfour ", Revue du Nouvel-Ontario, 15 (1993) : 70-71. Les activités minières régionales sont esquissées dans Carl Wallace et Ashley Thompson, dir., Sudbury. Rail Town to Regional Capital (Toronto, Dundurn Press, 1993); Gilbert A. Stelter, "Community Development in Toronto's Commercial Empire: The Industrial Towns of the Nickel Belt », Laurentian University Review, 6,3 (juin 1974) : 3-53; Noel Beach, "Nickel Capital: Sudbury and the Nickel Industry, 1905-1925», Laurentian University Review, 6,3 (juin 1974) : 55-74.

4. Les limites de l'ex-municipalité de Vallée-Est ne correspondent pas exactement à ces cantons. Mais le choix de six cantons entiers s'avère fort commode, compte tenu du mode de découpage des données gouvernementales.

5. À notre connaissance, ces registres n'ont d'équivalent ni au Québec ni au NouveauBrunswick. 
CART E 1

\section{Localisation des cantons examinés, région de Sudbury}

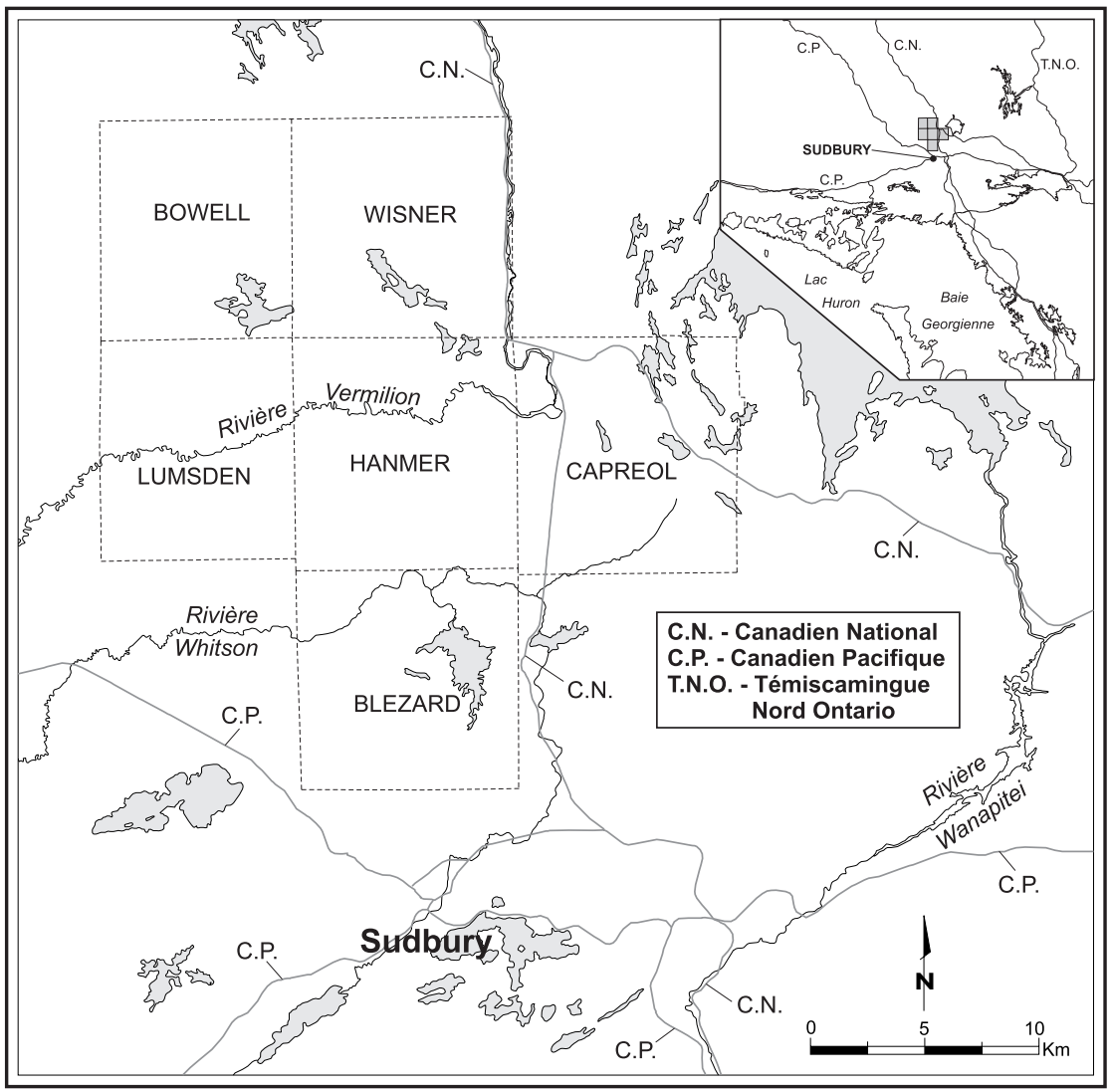

Réalisation : L.L.Larivière (Géographie, Université Laurentienne)

La richesse de cette documentation est telle qu'il convient de faire, dans une première partie, une courte présentation des principales sources utilisées. Cette présentation, toutefois, est précédée par un bref survol historiographique et par quelques propos théoriques. En deuxième partie, une vue d'ensemble des récoltes de matière ligneuse faites dans les six cantons sert d'introduction à deux analyses successives : d'abord, celle de la coupe dans les chantiers, grâce à l'examen des contremaîtres et des soustraitants, et ensuite celle de la coupe sur les lots agricoles. En conclusion, nous proposerons une évolution en trois étapes des activités forestières en milieu agricole. 


\section{HISTORIOGRAPHIE ET SOURCES UTILISÉES}

Depuis Arthur Lower ${ }^{6}$, les travaux des historiens sur la forêt ont plus souvent qu'autrement examiné les mécanismes des activités forestières dans un cadre géographique très large. D’abord perçue comme staple et pièce maîtresse de la thèse laurentienne pour expliquer le xix ${ }^{\mathrm{e}}$ siècle, la forêt est devenue un aspect incontournable de l'histoire régionale reconstituée dans une perspective sociale ${ }^{7}$. Chaque fois, les mécanismes retenus rendent compte d'entités globales comme les marchés, le cycle des produits forestiers (bois équarri, bois de sciage et bois à pâte), les politiques de gestion, d'importation ou d'exportation, les entrepreneurs en tant que groupe social ou encore la monopolisation des ressources. Utile, cette perspective met en évidence l'immensité des territoires de coupe.

Cette tendance lourde de l'histoire forestière nous a beaucoup influencé. Nos travaux antérieurs ont presque toujours retenu un cadre géographique provincial ${ }^{8}$. Au centre de ce choix reposait la question de l'épuisement des ressources qui suscite et conditionne une forte mobilité du capital et du travail. L’homme ne dépasse les contraintes géographiques qu'au prix de déplacements. L'examen de l'épuisement de la matière ligneuse ne peut pas être mené dans le seul cadre régional. Faire l'histoire d'une région - au demeurant utile pour rapprocher l'histoire universitaire des citoyens - nous est apparu souvent comme faire l'histoire des sédentaires, ces derniers étant valorisés par les élites, au détriment des sans-terre ou des migrants.

L'étude de la forêt à l'échelle locale a été largement négligée jusqu’à maintenant. Peut-être était-ce en raison du discrédit jeté sur les monographies paroissiales et la petite histoire, marquées par l'événement roi. Pourtant, comme nous le verrons, plusieurs éléments peuvent être mis en lumière grâce à cette nouvelle perspective.

À commencer par un examen des rapports entre l'agriculture et la forêt. Le concept d'économie agro-forestière développé brillamment par

6. Il paraîtra peut-être dépassé de nous référer aux travaux de Lower, publiés il y a déjà plus de 60 ans. Pourtant, ses ouvrages restent incontournables tellement la recherche sous-jacente était étoffée. Voir North American Assault on Canadian Forest (Toronto, Ryerson Press, 1938); «The Trade in Square Timber» (1933), dans W. T. Easterbrook et M. H. Watkins, dir., Approaches to Canadian Economic History (Toronto, MacMillan, 1978), 28-48.

7. Voir notamment René Hardy et Normand Séguin, Forêt et société en Mauricie (Montréal, Boréal, 1983). Les nombreuses monographies régionales parrainées par INRS-Culture (autrefois l'Institut québécois de recherche sur la culture) pourront être consultées à cet effet.

8. Notre dernier ouvrage l'illustre fort bien: Les récoltes des forêts publiques du Québec et de l'Ontario, 1840-1900 (Montréal, McGill-Queen’s University Press, 1999). 
Normand Séguin ne semble pas correspondre à la réalité des deux communautés de Hanmer et de Blezard ${ }^{9}$. Créer des surfaces cultivées et en vivre ont été rendus possibles en raison de la fertilité des terres et, au $\mathrm{xx}^{\mathrm{e}}$ siècle, de la proximité d'un grand centre urbain : Sudbury. Les activités forestières semblent y avoir laissé peu de traces et avoir peu dénaturé l'agriculture. En revanche, la notion de pluriactivité, mise en relief par Gérard Bouchard, paraît davantage prometteuse puisqu'elle retient les activités forestières comme une des dimensions du monde agricole avec lesquelles les agriculteurs composent ${ }^{10}$. Le travail dans les chantiers et la vente de produits forestiers seraient ainsi deux des multiples rapports que la paysannerie entretiendrait avec le capitalisme, les revenus accumulés servant avant tout à l'acquisition de terres additionnelles pour y établir les fils. La multiplication des dépendances déboucherait, en fin de compte, sur une relative autonomie des agriculteurs, faisant en sorte qu'aucune activité ne puisse prédominer et corrompre le travail de la terre.

Cette notion de pluriactivité impliquerait finalement que les activités forestières, comme toutes les autres, soient de courte durée et que le capitalisme tirerait profit de cette non-permanence de la relation. Seule condition pour que s'établisse une relation de complémentarité. Mais comment tout cela s'effectue-t-il? Nous croyons qu'un examen des activités forestières à l'échelle locale éclairera cette question. En effet, cette approche permet une lecture beaucoup plus fine et détaillée, tout en ouvrant d'intéressantes pistes de réflexion sur les différentes étapes du développement des activités forestières en milieu agricole.

Quelques mots maintenant à propos des sources. La première d'entre elles se compose des registres des concessions forestières ${ }^{11}$. Servant de source primaire à l'étude de la monopolisation des territoires de coupe, ces registres sont connus de plusieurs historiens ${ }^{12}$. Ils sont également utiles

9. Normand Séguin, La conquête du sol au 19e siècle (Montréal, Boréal Express, 1977); Normand Séguin, "Problèmes théoriques et orientations de recherche», dans Normand Séguin, dir., Agriculture et colonisation au Québec (Montréal, Boréal express, 1980), 181-197.

10. Gérard Bouchard, Quelques arpents d'Amérique (Montréal, Boréal, 1997); voir notamment le chapitre 6.

11. Ces documents légaux enregistrent le titulaire et la superficie de chacune des concessions forestières ontariennes. Ils sont déposés aux Archives publiques de l'Ontario à Toronto. Leur consultation est néanmoins difficile en raison du mode d'organisation des données. En effet, ces concessions - à l'inverse des québécoises — n'ont pas de code numérique constant, de sorte que reconstituer l'histoire de l'une d'elles peut s'avérer une tâche fort ardue.

12. Pour le Québec, voir notamment Serge Laurin, Histoire des Laurentides (Québec, Institut québécois de recherche sur la culture, 1989), 324 et ss; Camil Girard et Normand Perron, Histoire du Saguenay-Lac-Saint-Jean (Québec, Institut québécois de recherche sur la culture, 1989), 191 et ss; 
pour repérer les détenteurs des permis de coupe et, conséquemment, les distinguer des autres intervenants forestiers actifs dans une région, tels que les sous-traitants des grands travaux de construction, les marchands et les autres intermédiaires qui achètent du bois. C'est pourquoi leur utilisation ne peut être profitable qu'en les jumelant à une autre source gouvernementale, moins connue des historiens : les registres de coupe - et, dans le cas qui nous concerne, ceux de l'agent forestier du district de Sudbury. Nous les avons exploités systématiquement, ce qui nous a permis de mieux circonscrire les droits de coupe des différents intervenants forestiers. Ainsi y figurent quelques intermédiaires qui, sans être concessionnaires, doivent rendre compte à l'agent forestier des achats de bois effectués. En fait, si les registres des concessions donnent une image des droits théoriques des entreprises, les registres de coupe témoignent de leur mise en pratique.

Les registres de coupe, moins connus, nécessitent une présentation de leurs principales caractéristiques. Tenus depuis la saison de coupe 19011902, ils permettent à l'agent forestier de déterminer la somme des redevances forestières établie à partir de la déclaration annuelle de chacune des entreprises. Certes, ces déclarations volontaires sous-estiment considérablement les volumes de coupe. Il ne serait pas exagéré d'avancer que les volumes réels sont de $50 \%$ supérieurs à ceux officiellement enregistrés ${ }^{13}$. Mais comme ce défaut a toutes les chances d'affecter l'ensemble de la période, l'analyse proposée reste valable.

L'obligation de préciser la provenance du bois s'avère décisive dans notre démarche. Tout d'abord, elle permet d'identifier annuellement les coupes effectuées dans les six cantons rattachés aux deux communautés.

J. I. Little, Nationalism, Capitalism, and Colonization in the Nineteenth-Century Quebec (Kingston, McGill-Queen's University Press, 1989), chapitre 5. Pour les Maritimes, on consultera L. Anders Sandberg, dir., Trouble in the Woods. Forest Policy and Social Conflict in Nova Scotia and New Brunswick (Fredericton, Acadiensis Press, 1992); J. K. Hiller, "The Politics of Newsprint: The Newfoundland Pulp and Paper Industry, 1915-1939", Acadiensis, (printemps 1990) : 8 et ss. En Ontario, l'ouvrage récent de W. R. Wightman et Nancy M. Wightman, The Land Between: Northwestern Ontario Resource Development, 1800 to the 1990s (Toronto, University of Toronto Press, 1997), 182 et ss, illustre fort bien cette même utilisation des registres pour la cartographie des territoires de coupe.

13. Pour le Québec, nous avions tenté avec deux collègues de prendre la mesure de ce phénomène de sous-enregistrement; voir Guy Gaudreau, Claire-Andrée Fortin et Robert Décarie, "Les récoltes des forêts publiques (1850-1900) : proposition de correction des données", Revue d'histoire de l'Amérique française, 46,3 (hiver 1993) : 485-499. Notons, par ailleurs, que ce n'est pas tellement le nombre de billes de bois produites qui est systématiquement sous-estimé, mais les volumes physiques de ces mêmes billes qui sont beaucoup plus faciles à falsifier. 
Ensuite, elle s'accompagne de l'obligation de préciser si le bois a été cueilli sur des terres "patentées» ou pas. La distinction est essentielle, car les terres "patentées», pour reprendre l'expression usuelle de l'époque, désignent les terres qui sont pleinement la propriété des agriculteurs et qui, à ce titre, sont exclues du calcul des redevances qu'une entreprise doit verser pour le bois coupé sur les Terres de la Couronne. Il devient donc possible, grâce à cette distinction, de départager la coupe sur les lots agricoles de celle faite à l'intérieur des concessions forestières. Il arrivera, au besoin, que l'entreprise soit forcée de faire la preuve qu'elle peut être exemptée pour le bois provenant des terres "patentées», en identifiant le ou les lots où ce bois a été abattu. C'est que tous les lots agricoles n'étaient pas «patentés» de manière systématique. Plusieurs, en effet, ne l'étaient pas au moment de la coupe, car leurs détenteurs n'avaient pas respecté toutes les conditions pour bénéficier de la "patente».

\section{UNE VUE D'ENSEMBLE DE LA COUPE}

Le fait que la première saison d'exploitation inscrite dans le registre soit celle de 1901-1902 $2^{14}$ soulève une difficulté puisque le début des activités forestières de la région examinée est antérieur. Si l’on en croit les données relatives aux concessions forestières affermées dans les six cantons, c'est au milieu des années $1890^{15}$ que les entreprises obtiennent leurs premiers territoires de coupe ${ }^{16}$. Comme on peut le voir dans le graphique 1 , les surfaces concédées augmentent rapidement au tournant du $\mathrm{xx}^{\mathrm{e}}$ siècle. Mais étant donné que, d'une part, il y a généralement un certain délai entre l'obtention du permis de coupe et le début des activités d'abattage et que, d'autre part, un nombre encore plus considérable de concessions sont accordées après 1901, on peut raisonnablement avancer que les données obtenues dans le registre permettent d'examiner la plupart des activités forestières.

14. Pour les années antérieures, on pense que l'agent responsable était basé à Webwood, village situé à 50 kilomètres à l'ouest de Sudbury. Le registre de coupe qu'il aurait tenu n'a toujours pas été trouvé.

15. Les activités forestières sur la rive nord de la baie Géorgienne démarrent au cours des années 1880; voir Arthur Lower, North American..., op. cit., 176-179. Mais on s'attaque d'abord aux principaux bassins, tels que ceux des rivières Spanish et des Français. Les eaux qui coulent dans les six cantons sont des tributaires de la rivière Spanish, très éloignées de l'embouchure située plus à l'ouest.

16. Archives publiques de l'Ontario, Ontario Crown Lands, Registre des concessions forestières de l'Ontario, Western District, 6 (1894-1914) : concessions 6W105, 6W534, 6W574, 6W575, 6W583, 6W593, 6W584, 6W591, 6W5926W613, 6W675, 6W850; 7 (1907-1950) : concessions 7W27, 7W82. 
G RAPHI Q U E 1

Surfaces affermées, 1896-1921, en $\mathrm{mi}^{2}$

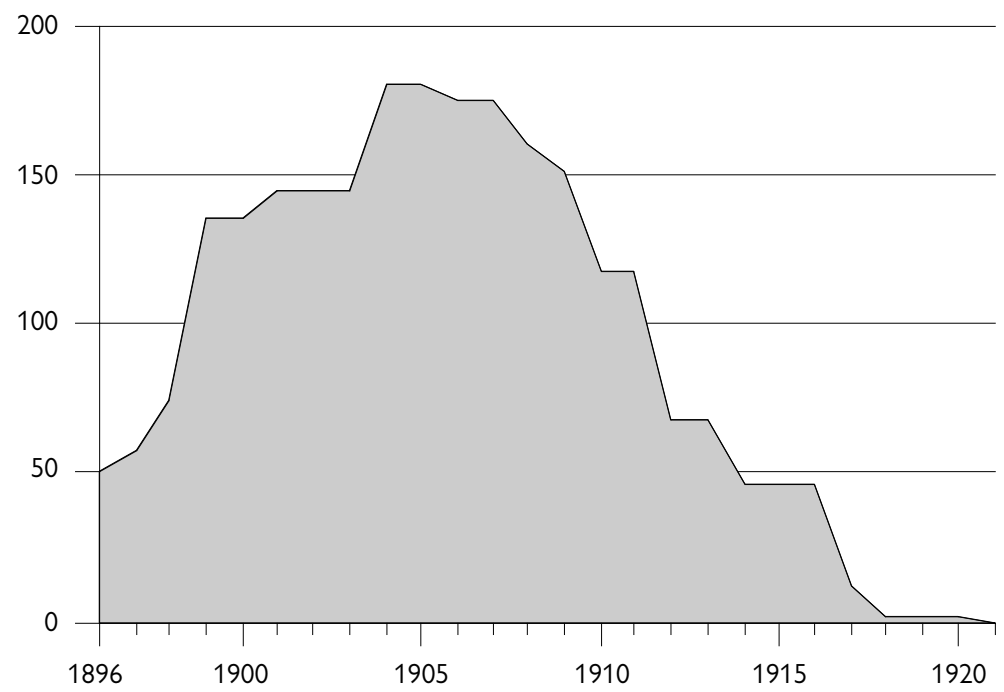

Nombreuses sont les entreprises titulaires de concessions forestières, notamment parce qu'elles sont souvent de faible superficie. Le canton de dimension régulière s'étend sur 36 milles carrés et peut être subdivisé pour former quatre concessions, comme c'est le cas des cantons de Hanmer et de Capreol. Une autre raison explique ce foisonnement : la nouvelle règle d'affermage des concessions en vigueur depuis 1892, à partir de laquelle les autorités gouvernementales peuvent céder concurremment un permis pour le bois de sciage et un autre pour le bois à pâte dans le même canton. Cette mesure, dont les historiens ont du mal à saisir toutes les répercussions $^{17}$, autorise l'octroi, à la Spanish Pulp and Paper, d'un vaste permis de coupe qui inclut quatre des six cantons de la zone étudié ${ }^{18}$ tout en

17. Nous avons amorcé la réflexion au sujet des répercussions de cette mesure sur la soustraitance forestière qui profite de la présence de deux entreprises. Voir Guy Gaudreau, «La soustraitance forestière dans le Nord-Est ontarien, 1900-1930 ", Labour/Le Travail, 40 (automne 1997) : 75-112. Nous avons également examiné son impact environnemental, étant donné que cette mesure accélère le rythme d'exploitation des ressources. Voir Idem, "L’importance du cadre juridique dans les approvisionnements de bois : le bassin de la rivière Sturgeon, 1898-1918 ", dans François-Xavier Ribordy, dir., La nature et la loi (Sudbury, Presses de l'Université Laurentienne, 1999), 227-241.

18. En fait, le contrat d'approvisionnement que prescrit la concession papetière (pulpwood concession) demeure assez vague en ce qui concerne les cantons dans lesquels l'entreprise exerce 
permettant au ministère des Terres de la Couronne de vendre les droits sur les pins aux entreprises de sciage.

La fin des concessions forestières, à compter des années 1910, ne provoque pas sur-le-champ le départ des entreprises forestières. La Spanish s'approvisionne directement auprès des agriculteurs pendant quelques saisons. Mais plus important encore, des entreprises locales, telles les sociétés Haight \& Dickson ou McCreary \& Graham, prennent le relais des concessionnaires en rachetant des restes de concessions ou en sillonnant la campagne, à l'affût de matières ligneuses pouvant encore être commercialisées. C'est dans ce contexte que s'inscrit l'arrivée de petites compagnies acheteuses de bois, fréquemment rencontrées dans les registres de coupe.

Comme on peut le voir dans le graphique 2, plusieurs catégories de bois ont fait l'objet de coupes ${ }^{19}$. Leur évolution respective semble être marquée par une certaine logique où les essences nobles, comme les pins blancs et rouges ${ }^{20}$, sont les premières à tomber, suivies par le bois à pâte, puis par le bois divers qui, ici, est composé de deux produits principaux, à savoir les traverses de chemin de fer et le bois de chauffage. La succession des catégories est davantage visible dans le graphique 3 .

La surprise de cette évolution demeure sans doute la forte présence de bois divers ${ }^{21}$. À l'échelle locale, ils peuvent constituer une part importante des abattages en raison d'une conjoncture particulière. Ainsi, la construction du chemin fer Canadien Nord ${ }^{22}$, qui sillonne les cantons de Capreol et de Lumsden entre 1906 et 1908, génère une forte demande de traverses que les entrepreneurs de la région s'empressent de fournir. Un autre dé-

un permis de coupe. Voir les Documents de la Session de l'Ontario, 1900, XXXII,10, "Agreement Between Her Majesty and the Spanish River Pulp and Paper Company... September 21, 1899", 50, 5. C'est une lecture attentive des registres de coupe qui nous a permis de déterminer très précisément les cantons inclus dans l'entente de 1892.

19. Ces données proviennent des Archives publiques de l'Ontario, Ontario Crown Lands, RG 144-0-11, Timber Agent's Book of the Sudbury District, 1901-1975, 1 (1901-1923), 298 p.

20. L'arpenteur chargé d'examiner le canton de Hanmer à l'été 1894 avait bien vu les valeurs marchandes que représentaient ces pins : "The township is well timbered, having white and red pine of a fair size and good quality...». Documents de la Session de l'Ontario, Rapport du commissaire des Terres de la Couronne, 1895(?), «Surveyors Report», cité dans Huguette Parent, Le Township de Hanmer..., op. cit., 45.

21. Cette observation n'est pas sans rappeler le commentaire de Douglas McCalla à propos de l'importance de ces "autres catégories de bois»; voir «Forest Products and Upper Canada Development, 1815-1846", Canadian Historical Review, 68,2 (1987) : 159-198.

22. Gaétan Gervais, «Le réseau ferroviaire du nord-est de l'Ontario, 1881-1931», Revue de l'Université Laurentienne, 13,2 (février 1981) : 55. 
GRAPHIQUE 2

Catégories de bois récoltés, $1901-1918$, en 0000 pi $^{3}$

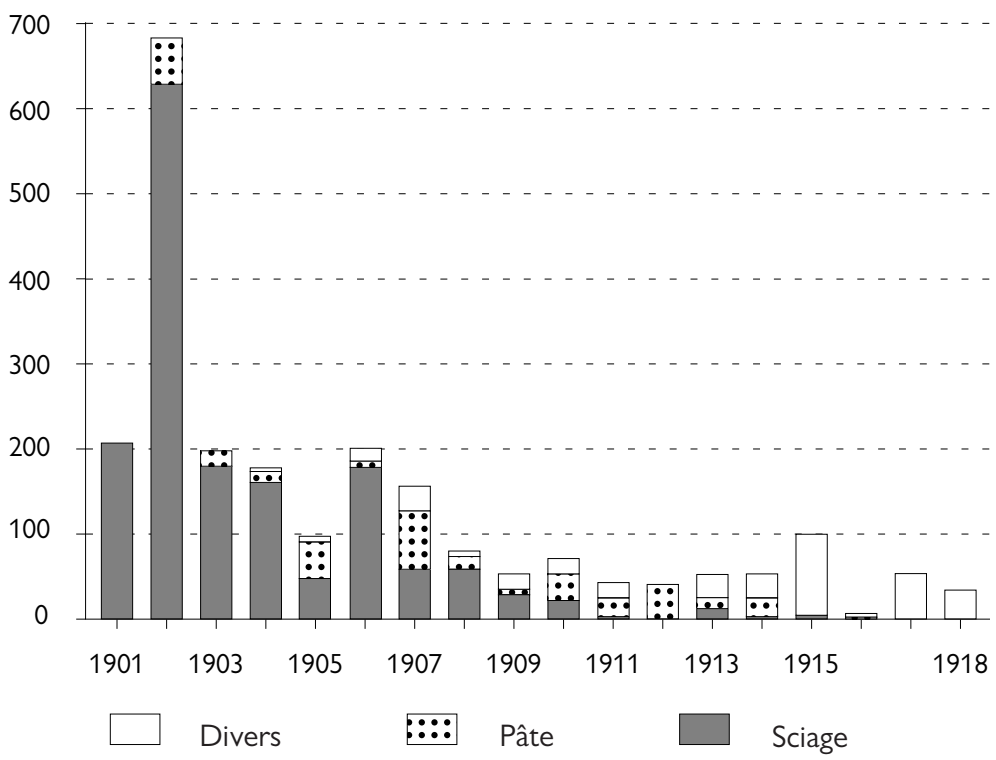

GRAPH I Q U E 3

Catégories de bois récoltés, 1901-1918, en \%

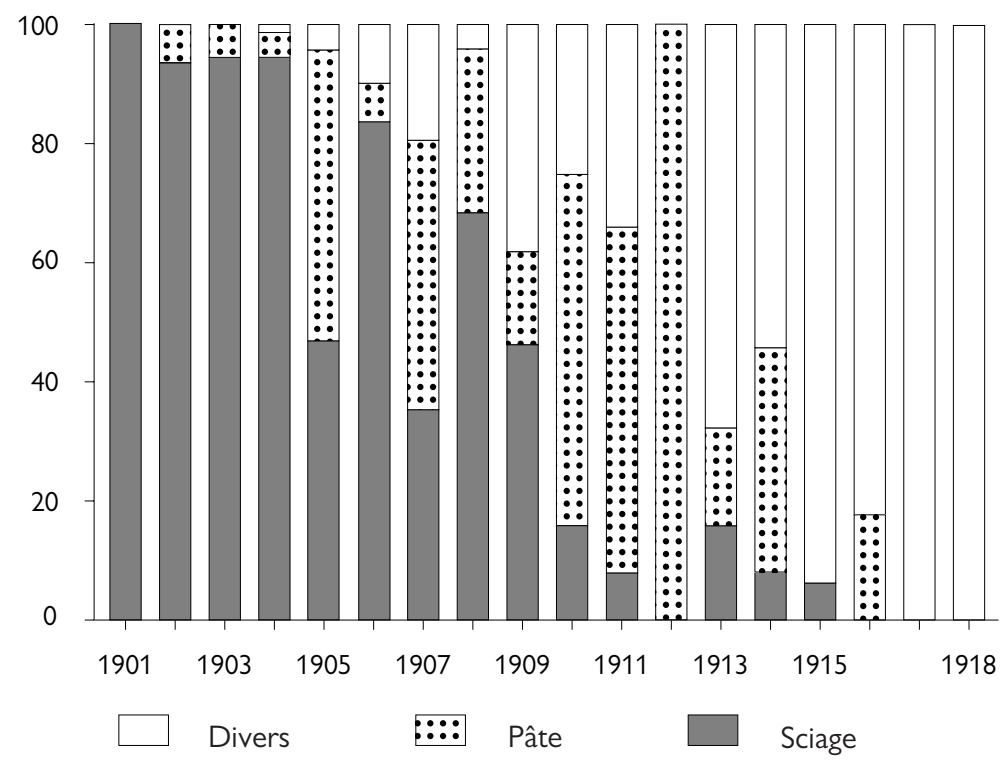


bouché vient des compagnies minières qui, au cours de la Première Guerre mondiale, accroissent considérablement leur production et, conséquemment, leur besoin de bois de chauffage afin d'alimenter les lits de frittage $^{23}$. Ce sont des dizaines de milliers de cordes de bois qu'elles engouffrent chaque année, ce qui vide la campagne des bois encore debout.

Une autre surprise demeure peut-être le volume total récolté lors de la saison 1902-1903. Dans le seul canton de Capreol — affermé pour la première fois en 1899 - on récolte près de 20 millions de $\mathrm{pmp}^{24}$ et 4000 cordes de bois à pâte, soit à peu près les niveaux de production annuelle enregistrés pour l'ensemble de la région du Saguenay-Lac-Saint-Jean à la fin du $\mathrm{XIX}^{\mathrm{e}}$ siècle. Difficile de trouver comparaison plus marquée! Il y a certes épuisement des ressources commerciales dans les forêts québécoises du dernier quart du $\mathrm{xIX}^{\mathrm{e}}$ siècle $^{25}$, mais il y a aussi, dans la région que nous étudions, abondance de pins majestueux ${ }^{26}$. De telles quantités, quoique nettement au-dessus d'une récolte moyenne, ne paraissent pas exceptionnelles à la lumière des abattages d'un canton limitrophe comme Lumsden ou de celui, un peu plus éloigné, de Morgan.

Comme ces données représentent l'ensemble des récoltes des intervenants forestiers, il convient de les répartir selon le statut juridique des boisés concernés. Notons au préalable que les chantiers forestiers à l'intérieur des concessions forestières englobent les productions des contremaîtres et celles des sous-traitants, sur lesquelles nous nous arrêterons plus loin.

Comme l'indiquent les graphiques 4 et 5 , la part des concessions forestières domine largement les récoltes ${ }^{27}$, sauf entre 1907 et 1911, alors que

23. Ces lits de frittage font quelques dizaines de mètres de largeur par, peut-être, une centaine de mètres de longueur. On les recouvre d'une bonne épaisseur de bois pour ensuite y déposer le minerai fraîchement hissé à la surface afin de le griller, éliminant ainsi une partie du soufre qu'il contient. La combustion dure ainsi quelques semaines, voire quelques mois, et provoque des fumées sulfureuses qui ont beaucoup endommagé la végétation régionale.

24. Un pmp (c'est-à-dire "pied mesure de planche»), équivaut à 144 po $^{3}$ de bois.

25. Camil Girard et Normand Perron nous avaient reproché cette affirmation relative à l'épuisement des ressources ligneuses saguenayennes qu'ils disaient trop considérables pour subir aussi rapidement un tel sort (C. Girard et N. Perron, Histoire du Saguenay..., op. cit., 609, note 18). Précisons que, dans notre esprit, cet épuisement est relatif puisqu'il concerne les spécimens alors commercialisés et générateurs de profits; voir G. Gaudreau, Les récoltes..., op. cit., chapitre 7.

26. Un collègue, Léo Larivière, nous a montré une souche d'un de ces pins du canton de Capreol. Vraisemblablement coupé au début du siècle, cet arbre avait, à sa base, un diamètre de plus d'un mètre, ce qui corrobore l'ampleur des volumes inscrits dans les registres.

27. Notons que certaines récoltes de faible volume ont été exclues du total des concessions forestières pour être attribuées aux lots agricoles. Ces cas nous ont semblé être le résultat du manque de précision quant au lot impliqué. 
la construction du chemin de fer se termine et que la demande de bois à pâte est soutenue. S'il y a remontée de la coupe dans les concessions forestières avec la Première Guerre mondiale, on la doit au canton de Wisner, plus inaccessible et moins pourvu en bois de sciage mais doté d'essences vulgaires idéales pour les lits de frittage des sociétés minières.

La distinction entre concessions forestières et terres agricoles, quoique commode, gomme des différences importantes à l'intérieur de chacune de ces catégories en ce qui concerne la nature et la taille des intervenants. L'accès à des ressources ligneuses considérables à l'intérieur des concessions, ou restreintes au sein de petits lots agricoles, ne conduit pas obligatoirement à la présence de gros producteurs d'un côté ou de petits de l'autre. Des nuances surgissent, à commencer par celles qui apparaissent dans les chantiers forestiers.

\section{CONTREMAÎTRES ET SOUS-TRAITANTS}

Une première façon d'analyser les coupes annuelles enregistrées à l'intérieur des concessions est de les répartir selon les volumes déclarés. Au total, 55 individus se partagent l'équivalent de 77 coupes annuelles. De

GRAPHIQUE 4

\section{Récoltes selon le statut juridique des forêts, en 0000 pi $^{3}$}

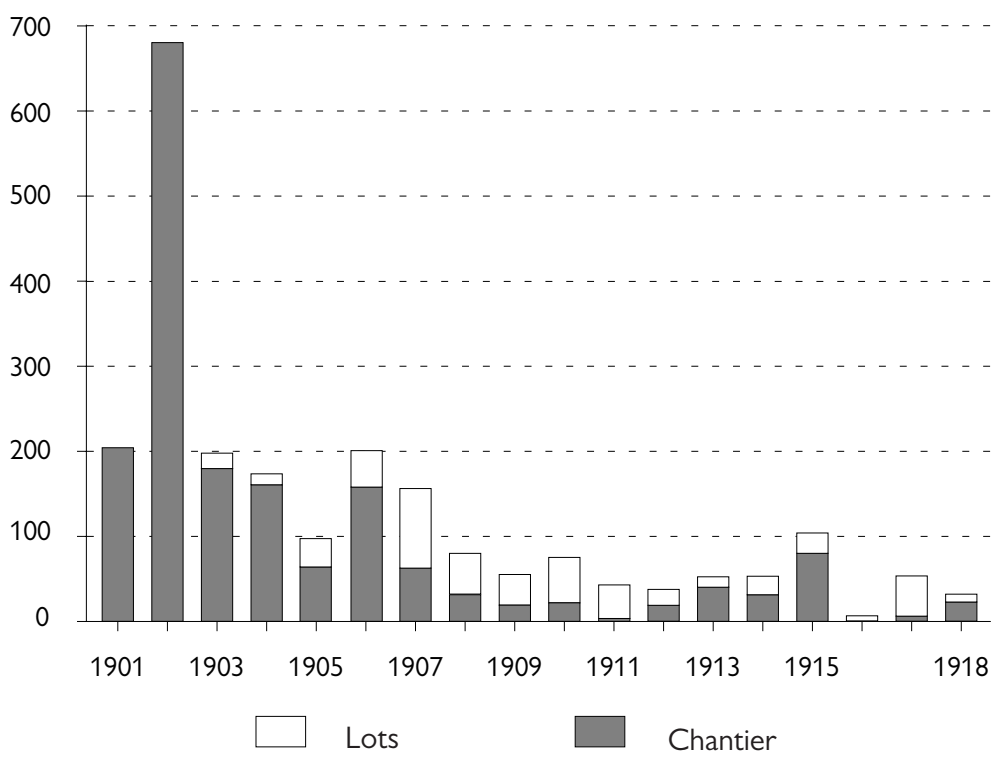


GRAPHIQUE 5

Récoltes selon le statut juridique des forêts, en \%

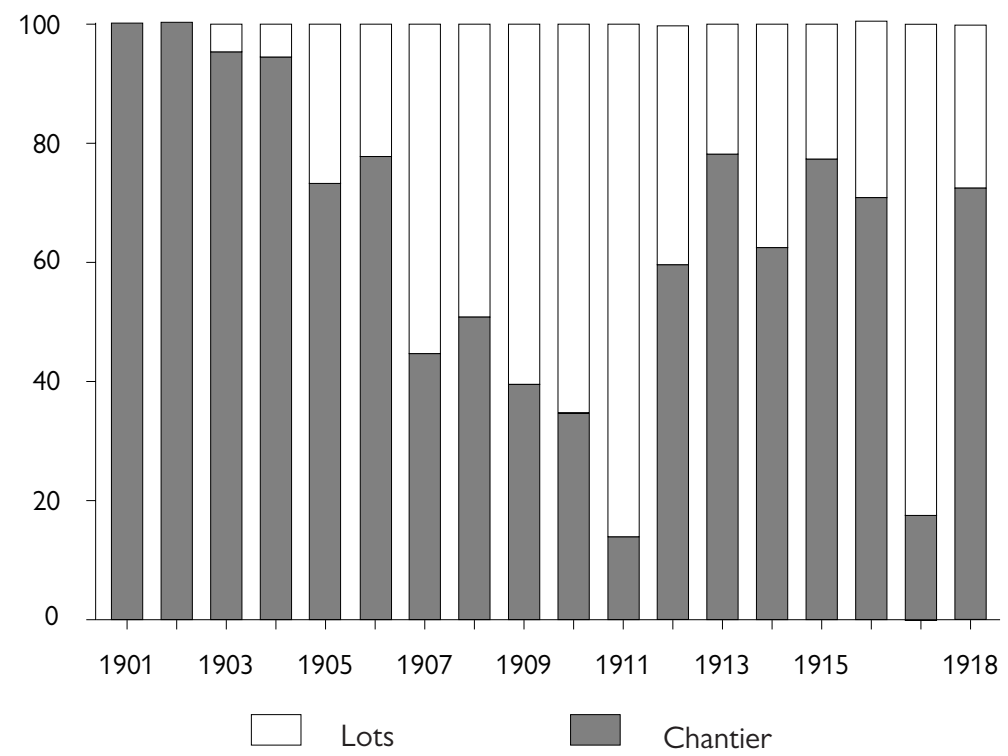

toute évidence, ces responsables des chantiers forestiers ne demeurent guère longtemps dans la région. Un seul, A. Dénommé, travaille pendant quatre saisons, d'ailleurs consécutives. L'individu typique fait une seule apparition. Quant aux Canadiens français, si l'on en croit la consonance des noms de famille, ils composent environ la moitié des effectifs.

Première constatation, $45 \%$ des chantiers ont une récolte inférieure à un million de pmp (voir le graphique 6). Afin de donner une vue plus concrète de ce million de pmp, retenons qu'il nécessite, selon les registres mêmes de l'agent forestier, la coupe de 10000 à 30000 billots (généralement de pin), dépendamment de la taille et du diamètre des spécimens. Autrement dit, un billot contiendrait en moyenne entre 35 et 100 pmp, ce qui est bien peu et ce qui démontre de belle manière le laxisme des autorités gouvernementales à l'égard des entrepreneurs forestiers. Ce sont plutôt entre 150 et 200 pmp que nous aurions dû trouver pour les pins blancs ${ }^{28}$.

28. L'écart entre la déclaration et les volumes réels est tel qu'on pourra douter de notre analyse. Il faut comprendre que les gestionnaires des forêts publiques de cette époque ne cherchent pas à connaître les volumes exacts. Ils tolèrent une situation presque séculaire qu'ils ne pourraient changer sans bouleverser radicalement les rapports avec les barons du bois. 


\section{GRAPHIQUE 6}

\section{Les chantiers selon les volumes en 0000 pmp, 1901-1918}

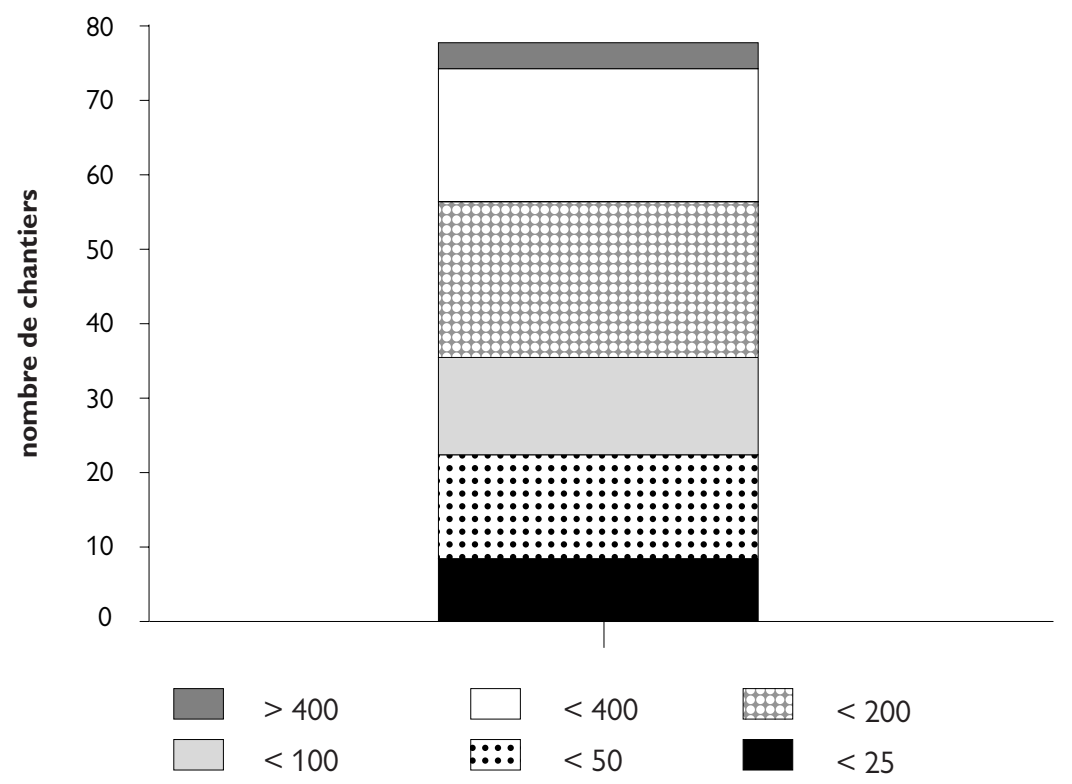

Une autre façon commode de se représenter un million de pmp est de le traduire en besoin de main-d'œuvre. Très grossièrement, on peut estimer que chaque million de pmp requiert l'équivalent de dix hommes pendant six mois, sans compter la drave ${ }^{29}$. Ce nombre comprend cependant les bûcherons ainsi que les charretiers.

Puisque près de la moitié des chantiers observés produisent moins d'un million de pmp, ils sont en moyenne de petite taille. Cela ne doit pas nous surprendre car le phénomène a été observé au Québec et ailleurs dans le Nord-Est ontarien $^{30}$. De petits chantiers impliquent vraisemblablement des constructions temporaires et mal chauffées, de même que des conditions de travail précaires. Notons cependant que les chantiers produisant plus de deux millions de pmp forment le quart des effectifs. Ici, les camps sont mieux organisés et la division du travail y est peut-être plus fine. Dans les plus gros camps, on compte peut-être jusqu'à une cinquantaine d'hommes.

29. G. Gaudreau, Les récoltes..., op. cit., 137 et plus particulièrement la note 13.

30. Voir R. Hardy et N. Séguin, Forêt et société..., op. cit., 32 ; G. Gaudreau, «La sous-traitance...", op. cit., 87-90. 
Le recensement nominatif de 1901 fournit quelques indications à propos des chantiers forestiers. Bien qu'aucun camp n'ait été retrouvé dans les six cantons à l'étude - en raison d'une énumération tardive ${ }^{31}$ - une dizaine de camps ont été repérés plus à l'ouest et au sud. Cinq d'entre eux comptent moins de dix travailleurs. Quatre ont entre dix et dix-sept travailleurs, tandis que deux dépassent la vingtaine. Ainsi, ces données fragmentaires concordent assez bien avec l'image que projettent les données des registres de coupe. Le plus important abrite 27 travailleurs. Le plus petit compte quatre travailleurs et aucun cuisinier, de sorte qu'il s'agit vraisemblablement d'un campement temporaire. Par ailleurs, l'absence d'un responsable de chantier, dans trois cas sur onze, pourrait laisser croire que certains camps sont rattachés à d'autres et pourraient peut-être constituer des camps plus importants. Comme l'enregistrement se fait en avril, ces camps sont rendus à l'étape du transport du bois ou encore à celle de la drave.

L'image d'ensemble tirée de ces listes nominatives offre peu de surprise. Sur les 140 hommes recensés, $80 \%$ sont célibataires. Ce célibat ne s'explique pas seulement du fait qu'ils sont jeunes et fils : en effet, moins de la moitié de ceux dont l'âge est connu ont 25 ans et moins. Les travailleurs reconnus comme spécialisés sont peu nombreux ${ }^{32}$ : une dizaine de cuisiniers, quatre commis, trois mesureurs, quelques charpentiers. Les autres sont désignés sous le vocable de journaliers forestiers (shanty labourer) ou encore de journaliers (general labourer).

Fait à noter, il n'y a pas d'homogénéité ethnique. Tous les camps embauchent à la fois des Canadiens français et des Canadiens anglais, ces derniers étant légèrement majoritaires. Ce mélange exclut la possibilité que les travailleurs soient issus de la même paroisse. Même le responsable canadien-français du plus gros camp ne dirige qu'une dizaine de Canadiens français sur les 26 ouvriers formant ses effectifs. Les responsables de camp sont présentés, dans le recensement, comme des contremaîtres (foreman). Vu que cette terminologie est utilisée couramment à l'époque dans tous les secteurs économiques, il est difficile de savoir s'ils sont les employés d'une entreprise forestière ou s'ils sont des sous-traitants. Néanmoins,

31. Par exemple, les cantons de Capreol et de Wisner ont été visités en juin (et étaient inhabités), celui de Hanmer en mai.

32. Comme l'a bien démontré Ian Radforth, le travail de bûcheron et de charretier requiert une expertise qui les qualifierait facilement de travailleurs spécialisés. Les critères de classement en ont néanmoins décidé autrement : I. Radforth, Bushworkers and Bosses: Logging in Northern Ontario, 1900-1980 (Toronto, University of Toronto Press, 1987). 
l'absence d'homogénéité ethnique nous incite à croire qu'il s'agit de contremaîtres salariés de camps de compagnies forestières, même si la taille réduite de certains camps les rangerait du côté des sous-traitants.

Pour en savoir un peu plus long sur cette question des contremaîtres et des sous-traitants ${ }^{33}$, nous avons isolé, dans les registres de coupe, l'entreprise de sciage qui a récolté le plus de bois dans les cantons étudiés et nous l'avons suivie dans ses déplacements à l'intérieur du district de Sudbury. La société Holland \& Graves (elle porta également le nom de GravesBigwood) demeure active six saisons de suite dans les cantons étudiés, puis obtient de nouvelles concessions forestières plus à l'ouest. Nous savons peu de chose à son sujet, si ce n’est que sa scierie est installée à Byng Inlet, dans la baie Géorgienne ${ }^{34}$.

En quinze saisons d'exploitation, soit de 1901 à 1915, l'entreprise confie ses chantiers à 47 individus différents, dont 13 dans les 6 cantons étudiés. La grande majorité d'entre eux travaillent pendant un court laps de temps : 28 ne font qu'une saison (60\%), et 12 autres restent entre 2 et 3 ans ( $25 \%)$. Quatre autres individus reviennent dans les registres pendant quatre ou cinq ans. Trois seulement reviennent régulièrement. Il s'agit de William Beasley, qui suit l'entreprise pendant douze ans, Robert Brazil, qui compte sept années de service, et Alfred Lewis, qui n'est pas en reste avec neuf saisons.

À première vue, il pourrait s'agir de deux groupes d'individus : d'un côté, les plus stables qui restent fidèles à l'entreprise et qui ont peut-être le statut de contremaître et, de l'autre, des individus de passage qui ont plus de chance d'être des sous-traitants. Afin d'éclairer cette hypothèse, il convient néanmoins de mieux comprendre la mécanique de coupe d'une entreprise.

Le premier élément à mettre en évidence est sans conteste la courte durée de l'exploitation d'une concession forestière. Que la Holland \& Graves ne s'active dans la région étudiée que six années ne doit pas surprendre. Les autres titulaires de permis de coupe dans les cantons ne demeurent pas plus longtemps ${ }^{35}$. Seule la Spanish River Pulp \& Paper, qui détient des permis dans quatre des six cantons, s'affaire pendant treize années. Et cette mobilité des entreprises n’a rien de spécifique à la zone à l'étude. En effet, l'examen de deux entreprises régionales à l'œuvre pen-

33. L'ambiguité de l'historiographie sur la question de la sous-traitance a été explorée dans G. Gaudreau, «La sous-traitance...», op. cit., 75-77.

34. Denis Laforge, «L'industrie forestière dans le Nord-Est ontarien avant 1900 », dans Aspects du Nouvel-Ontario au XIX ${ }^{e}$ siècle II (Sudbury, Société historique du Nouvel-Ontario, 1981), 11.

35. Haight $\&$ Dickson s'active pendant 6 ans, McCreay $\&$ Graham 4 ans, Robert Booth 4 ans et Loveland \& Stone 3 ans. 
dant des décennies dans les districts de Sudbury et de North Bay, soit les entreprises de la famille Gordon et celle de Willam Milne \& Sons, donne des résultats assez semblables. Sur les 18 cantons dans lesquels ils ont été titulaires de droits de coupe, leurs récoltes, même les plus infimes, dépassent à peine 8 ans en moyenne. En somme, on retiendra que les entreprises déménagent régulièrement leurs chantiers forestiers, afin d'accéder à de nouvelles sources d'approvisionnement. C'est là une constante fondamentale de leurs activités.

La fluctuation des niveaux de coupe constitue une deuxième caractéristique. Des hausses et des baisses de récoltes de $50 \%$ ne sont pas rares. À l'occasion, la récolte peut même doubler d'une saison à l'autre. Ces fluctuations fréquentes sont causées par différents facteurs tels que les fluctuations du marché, les fréquents incendies de scieries, les conditions printanières (qui n'auraient pas permis, par exemple, la descente de toutes les billes la saison précédente) ou encore les conditions hivernales (une neige tardive ralentit le transport des billes).

Mais comme les chantiers ont des capacités maximales de coupe, cela a pour conséquence — il s'agit d'une dernière caractéristique — de faire fluctuer considérablement le nombre de camps ouverts d'une année à l'autre. Et cette variation du nombre de camps est aussi tributaire de la topographie de la concession et plus spécifiquement de la présence de lacs et de cours d'eau.

Tous ces facteurs concourent à ce que l'entreprise retienne finalement les services de deux catégories de gestionnaires de camp : ceux, peu nombreux, qui ouvrent la coupe dans une nouvelle concession forestière, qui s'attaquent aux plus beaux spécimens et qui gèrent de gros camps pour ensuite se déplacer vers la nouvelle concession forestière; puis ceux qui, selon les besoins du marché et des profits, viennent nettoyer la vieille concession en s'attaquant aux boisés de seconde qualité ou difficiles d'accès et qui peuvent être remerciés de leur service dès l'année suivante.

Certes, le premier groupe s'apparente davantage aux contremaîtres dont on peut aisément prévoir le rendement, et le deuxième aux soustraitants qui garantissent une rentabilité, même dans les boisés les plus difficiles $^{36}$. Nous conservons néanmoins cette impression qu'il s'agit d'une

36. Pierre Ouellette, qui a eu accès aux archives de la Spruce Falls Pulp \& Paper de Kapuskasing, a bien démontré la présence simultanée de ces deux catégories de chantiers forestiers. Voir P. Ouellette, «La mécanisation des activités forestières dans la région de Kapuskasing, 1928-1980 ", mémoire de maîtrise (histoire), Université Laurentienne, 1994, 96-97. 
démarcation pas toujours bien tranchée ${ }^{37}$. Il n'est pas impossible qu'un sous-traitant efficace se voie offrir la gestion d'un camp, la saison suivante, à titre de contremaître, tandis qu'un contremaître, de son propre chef, quitte un employeur exigeant ou en faillite pour se retrouver sous-traitant l'année suivante. N'oublions pas qu'à cette époque, on fait encore rarement carrière au sein d'une société forestière et que les entreprises enregistrent de fréquentes faillites ${ }^{38}$. En outre, il arrive parfois que des contremaîtres de chantier soient engagés par les gros sous-traitants qui sont nombreux à faire affaire avec les papetières ${ }^{39}$.

Il faut dire que la sous-traitance ne nous apparaît pas être, à court et à moyen termes, synonyme de prolétarisation urbaine vainement repoussée, d'échec économique et de misère rurale, comme elle est parfois dépeinte. Elle épouse parfaitement la pluriactivité du monde rural. Tantôt dans les chantiers, tantôt hors des chantiers à faire différents boulots. «La famille paysanne a réussi à réaliser une forme d'autonomie [...] au gré d'un fractionnement de ses dépendances (dans la pluriactivité) ${ }^{40}$.»

Nous l'avons démontré dans un texte antérieur, la sous-traitance, en Ontario, profite occasionnellement d'un système d'attribution de permis de coupe qui multiplie le nombre d'entreprises forestières à l'intérieur des mêmes cantons, en attribuant séparément des permis pour le bois à pâte et pour le bois de sciage. C'est le cas dans 4 des 6 cantons étudiés, puisque 7 des 77 coupes annuelles menées sur des concessions forestières ont été le produit d'un double contrat d'approvisionnement. Le sous-traitant en tire profit en minimisant les coûts puisque le même camp peut travailler aux deux contrats.

Qui sont ces contremaîtres et ces sous-traitants? La réponse est difficile à trouver, car nous ne connaissons que leur nom, tel que le précisent les registres de coupe. Sont-ils des agriculteurs installés dans l'une des deux communautés? L'examen des activités de coupe sur les lots agricoles permettra d'éclairer cette question complexe.

37. Voir G. Gaudreau, «La sous-traitance...», op. cit., 111-112.

38. Ibid.

39. On lira à ce sujet le témoignage d’Émile Boudreau qui raconte «Un hiver dans les chantiers" au cours des années 1930 ; voir E. Boudreau, Un enfant de la grande dépression. Autobiographie (Montréal, Lanctôt éditeur, 1998), 169.

40. Gérard Bouchard, "Quelques arpents d'Amérique. Les tenants et les aboutissants d'une enquête ", Revue d'histoire de l'Amérique française, 50,3 (hiver 1997) : 422-423. 


\section{LA COUPE SUR LES LOTS AGRICOLES}

On l'a vu plus haut, la coupe faite sur les terres agricoles n'atteint pas le volume de celle des concessions forestières. Ce qui ne signifie pas que le nombre d'individus touchés soit modeste, loin de là. Comme il fallait s'y attendre, ces individus, identifiés dans les registres, sont nombreux : près de 350. Arborant souvent les mêmes noms de famille $e^{41}$, ils ne possèdent pas nécessairement le lot sur lequel la coupe est menée. Nous y reviendrons.

Une première façon de prendre la mesure de ces activités forestières est d'en examiner la fréquence parmi tous les agriculteurs de la région. Il suffit alors de comparer la liste des agriculteurs issus de la région avec celle des individus déclarant une récolte. Afin d'inventorier tous les agriculteurs régionaux, nous avons systématiquement dépouillé les données du bureau d'enregistrement du district de Sudbury, identifiant, pour chacun des cantons, les noms des premiers détenteurs des titres de propriété de chacune des terres. Comme il peut se passer plusieurs années avant l'obtention des titres, cet inventaire a été poursuivi jusqu'à la fin des années 1920, nous assurant ainsi que ceux qui auraient pu être actifs dans les registres de coupe ont eu la chance d'obtenir leur terre. Ces 287 individus forment en quelque sorte les pionniers de ces deux communautés.

Moins de la moitié de ces colons (49\%) ont été retrouvés dans les registres de coupe ; c'est beaucoup moins que ce à quoi on s'attendait. Les activités forestières directes, c'est-à-dire celles menées sur les lots ${ }^{42}$, ne paraissent pas déterminantes dans leur destinée. Cherchant à connaître les facteurs pouvant inciter les agriculteurs à y avoir recours, nous avons distingué ceux déjà présents lors du recensement de 1901 et ceux qui étaient dans la région avant que le registre de coupe du district soit ouvert (et qui auraient donc pu déclarer du bois dans le passé). Mais encore ici, la proportion ne change guère puisque 35 des 64 familles ( $55 \%$ ) présentes lors du recensement de 1901 n’ont pas été repérées dans les registres de coupe.

On peut ainsi avancer l'hypothèse que le fait d'avoir recours aux activités forestières directes augmente si l'individu arrive après 1901. Mais encore faut-il que le lot ait été défriché le moins possible. En effet, la consultation des titres de propriété du bureau nous a permis de dresser une seconde liste d'individus, regroupant ceux qui avaient acheté la terre d'un

41. On dénombre, par exemple, 7 Martel, 7 Carrière, 6 Gervais, 5 Ménard, 5 Brunet, 5 Labelle et 5 Saint-Germain.

42. En suivant cette distinction arbitraire, le travail dans les chantiers équivaudrait aux activités indirectes. 
premier propriétaire ${ }^{43}$. Nous avons retenu seulement ceux qui ne figuraient pas sur la liste des pionniers. Ils sont 54 au total. Or seulement le quart d'entre eux (13) se retrouve dans les registres de coupe. À la lumière de ces données, les activités forestières directes seraient liées, dans une certaine mesure, au défrichement : une terre en partie défrichée offre, bien sûr, moins d'occasions de vente de produits forestiers.

Mais les premiers défrichements ne constituent pas l'unique explication des activités directes de coupe. En effet, les registres doivent indiquer mais ils ne le font pas toujours - si la coupe est affranchie du paiement des redevances ou pas, autrement dit, si le bois est récolté sur des terres «patentées» ou non. Or les résultats indiquent clairement que la majorité des activités de coupe s'effectuent après que le lot a été "patentét4». Cela pourrait surprendre; pourtant nous y voyons une certaine logique. D'abord, la coupe précédant la "patente» est sujette à des redevances qui diminuent sans doute la part des profits. Ensuite, la coupe des premières années est consacrée à l'établissement sur le lot, de sorte que le bois a alors une valeur d'usage. Finalement, le fait de "patenter» une terre ne signifie pas que toute sa surface ait été déboisée : il y a de fortes chances que les arbres de seconde qualité y soient encore abondants. La vente de bois interviendrait ainsi dans un deuxième temps, à un moment plus propice, ce qui semble laisser une certaine marge de manœuvre à l'agriculteur.

Revenons aux 347 individus identifiés plus haut dans les registres de coupe. La situation la plus fréquente, près de $60 \%$, c'est la vente effectuée au cours d'une seule saison. Cela pourrait souligner, encore une fois, le faible rôle joué par les activités forestières directes dans le développement de la paysannerie et, conséquemment, le recours à d'autres formes de rapport avec le monde marchand.

Une bonne façon de se faire une idée sur ces paysans d'une seule récolte de bois consiste à examiner de plus près certains d'entre eux. Nous avons sélectionné à cet effet tous ceux dont le nom de famille commence par la lettre B, afin de constituer un échantillon. Quarante individus ont

43. Ces individus constituent, en quelque sorte, une deuxième génération. Cette terminologie, pas très adéquate, permet toutefois de les distinguer des pionniers.

44. Lynn Bélanger, dans son mémoire de spécialisation «L'activité forestière des agriculteurs de la Vallée, 1900-1930", (histoire, Université Laurentienne, 1999), estime qu’il y avait une légère domination des coupes sur les terres "patentées » dans les cantons de Balfour et de Lumsden (voir 34-36). Nos calculs, effectués à partir des agriculteurs plus actifs dans la vente de matière ligneuse dans les six cantons, indiquent plutôt une nette domination des terres "patentées». Par prudence, il convient de retenir une coupe très importante après l'obtention de la "patente ». 
été ainsi retenus dans les registres de coupe. Parmi eux, 24 ne font qu'une seule apparition : leur nom apparaît au tableau 1.

TAB LEA U

\section{Quelques caractéristiques des agriculteurs présents au cours d'une seule saison, 1901-1918}

\begin{tabular}{|c|c|c|c|c|}
\hline Nom & Saison* & $\begin{array}{c}\text { Produits forestiers } \\
\text { vendus }\end{array}$ & $\begin{array}{c}\text { Nombre } \\
\text { d'acheteurs }\end{array}$ & $\begin{array}{c}\text { Membres } \\
\text { des deux } \\
\text { premières } \\
\text { générations }\end{array}$ \\
\hline Baleau, Henry & 1910 & 1739 traverses & & non \\
\hline Balicki, W. & 1917 & 70 c. chauffage & & non \\
\hline Barbeau, J. & 1908 & 21 cordes & & non \\
\hline Bélanger, Joseph & 1907 & 300 cordes & & non \\
\hline Berthiaume, Francis & 1910 & 558 trav. 12 cordes ++ & 2 & non \\
\hline Bertrand, Gédéon & 1907 & 11 cordes & & non \\
\hline Bérubé, J. & 1918 & 160 c. chauffage & & non \\
\hline Bigras, Chap. & 1914 & 49 cordes & & non \\
\hline Billard, Philias & 1911 & 48 cordes & & non \\
\hline Bishop, Hary & 1911 & 57 cordes & & non \\
\hline Blais, D. & 1906 & 11 cordes & & \\
\hline Borey, Fred & 1906 & 174013 pmp & & non \\
\hline Boyer, O. & 1907 & 993 traverses & & non \\
\hline Briand, Peter & 1907 & 60 cordes & & non \\
\hline Brisebois, $\mathrm{A}$. & 1912 & 441 cordes & & non \\
\hline Brisebois, Édouard & 1911 & 59 cordes & & non \\
\hline Brosseau, Charles & 1913 & 200 cordes & & non \\
\hline Brosseau, L. & 1906 & 95356 pmp & & non \\
\hline Bruneau, G. & 1908 & 56 cordes & & non \\
\hline Brunelle, J.-Pierre & 1910 & 1030 traverses & & \\
\hline Brunet, Alphonse & 1905 & 61 cordes & & non \\
\hline Brunet, Joseph & 1907 & 1274 cordes & 2 & non \\
\hline Brunet, Victor & 1911 & 109 poteaux & & non \\
\hline Burdon, F. & 1911 & 8 cordes 18 poteaux & 2 & non \\
\hline
\end{tabular}

* Par commodité, nous n'avons identifié que l'année civile de l'automne de chaque saison d'exploitation.

** Une case vide indique l'affirmative. 
Tous, sauf trois, ont fait affaire avec une seule entreprise. Ils ont vendu principalement du bois à pâte. Les volumes récoltés restent faibles, c'està-dire à la portée d'une famille paysanne. Seul Joseph Brunet a produit un volume de coupe qui dépasse de beaucoup celle que fait une famille. S'agit-il d'une erreur? Pas nécessairement. Il pourrait être un sous-traitant à l'emploi de la Spanish Pulp \& Paper et qui s'est entendu avec un agriculteur pour faire chantier sur son lot. Cela dit, il ne faut pas croire que, même en faisant abstraction des sous-traitants, les volumes de coupe sur les lots soient toujours modestes. Finalement, un nombre élevé d'entre eux conserve un statut indéterminé. Ils ne font pas partie des pionniers et sont peut-être des colons de passage qui ont décidé de ne pas prendre racine dans la région.

Examinons maintenant ceux que nous retrouvons fréquemment dans les registres de coupe et que nous appelons «les agriculteurs actifs». La durée de leurs activités forestières dépasse celle de ceux qui travaillent à l'intérieur des concessions forestières puisque $10 \%$ d'entre eux enregistrent des ventes de bois pendant au moins quatre saisons. Nous avons voulu, ici encore, en savoir davantage en isolant, cette fois-ci, ceux qui ont fait des récoltes pendant quatre ans au minimum. Le tableau 2 synthétise l'information recueillie à leur sujet.

Ces 36 individus - dont les trois quarts font partie des deux premières générations ${ }^{45}$ - sont donc ceux qui ont davantage recouru aux activités forestières directes. Pourtant, en moyenne, leurs coupes commercialisées ne durent guère plus de cinq ans. Qui plus est, les saisons de travail sont rarement consécutives. En excluant les récoltes de bois de chauffage pour l'autoconsommation et, très certainement, des récoltes jamais déclarées, ces activités directes demeurent modestes, comparées à une quarantaine d'années de travail sur la terre. Cela mérite attention : production et vente de produits forestiers ne constituent qu'une activité parmi tant d'autres.

Cela dit, dressons un portrait de ces activités, à commencer par le fait que les agriculteurs font affaire avec plus d'une entreprise, malgré la courte durée de leurs activités. Cette observation n'est pas tributaire d'une méthodologie laxiste dans le calcul des entreprises. Des sociétés comme la compagnie de chemin de fer Canadien Nord et les différentes sociétés soustraitantes concernées par la construction de son réseau ont été comptées

45. Et encore cette proportion est au-dessous de la réalité, car cinq des neuf individus n'apparaissant pas sur la liste des pionniers partagent néanmoins un nom de famille inscrit sur cette liste. 


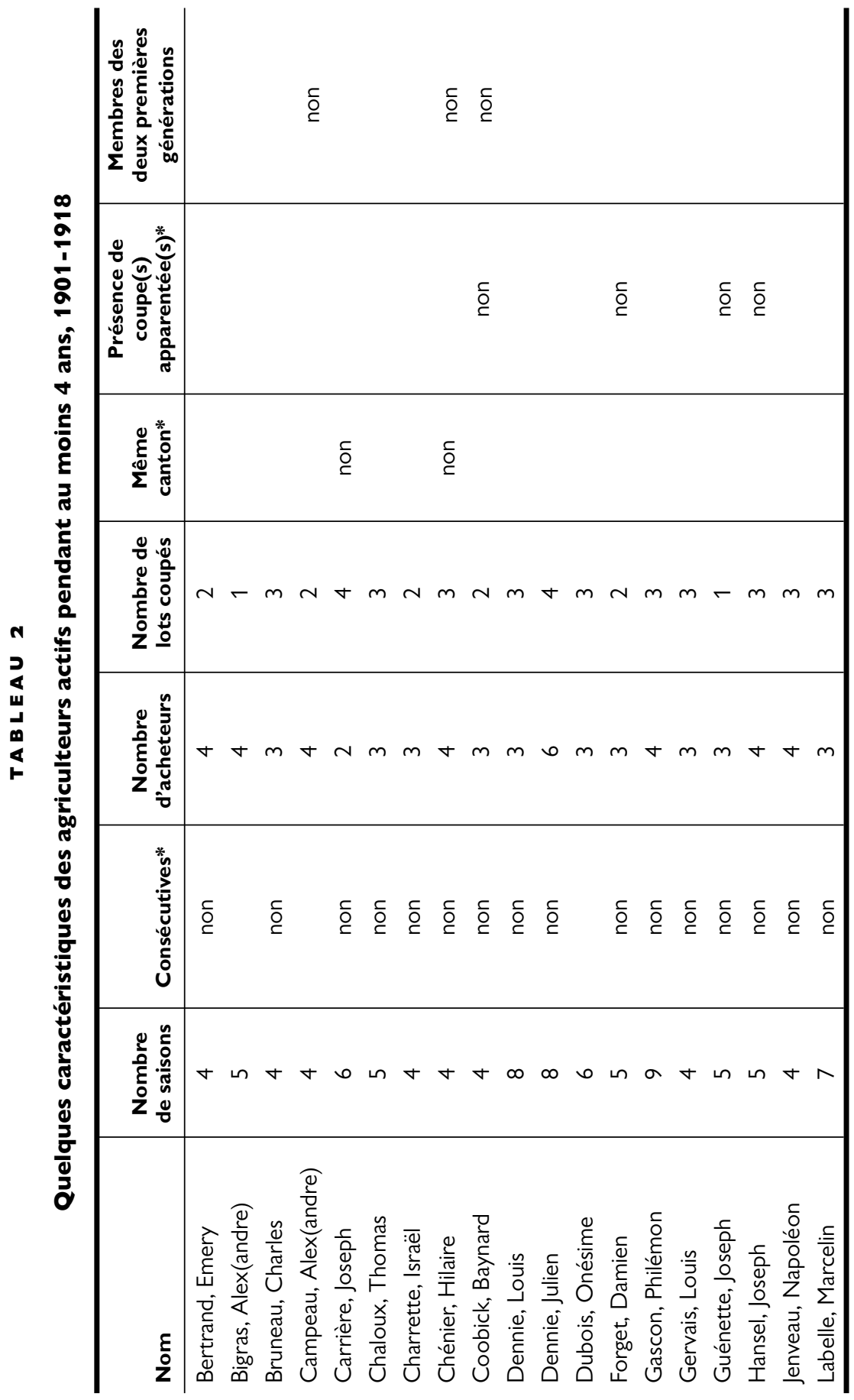




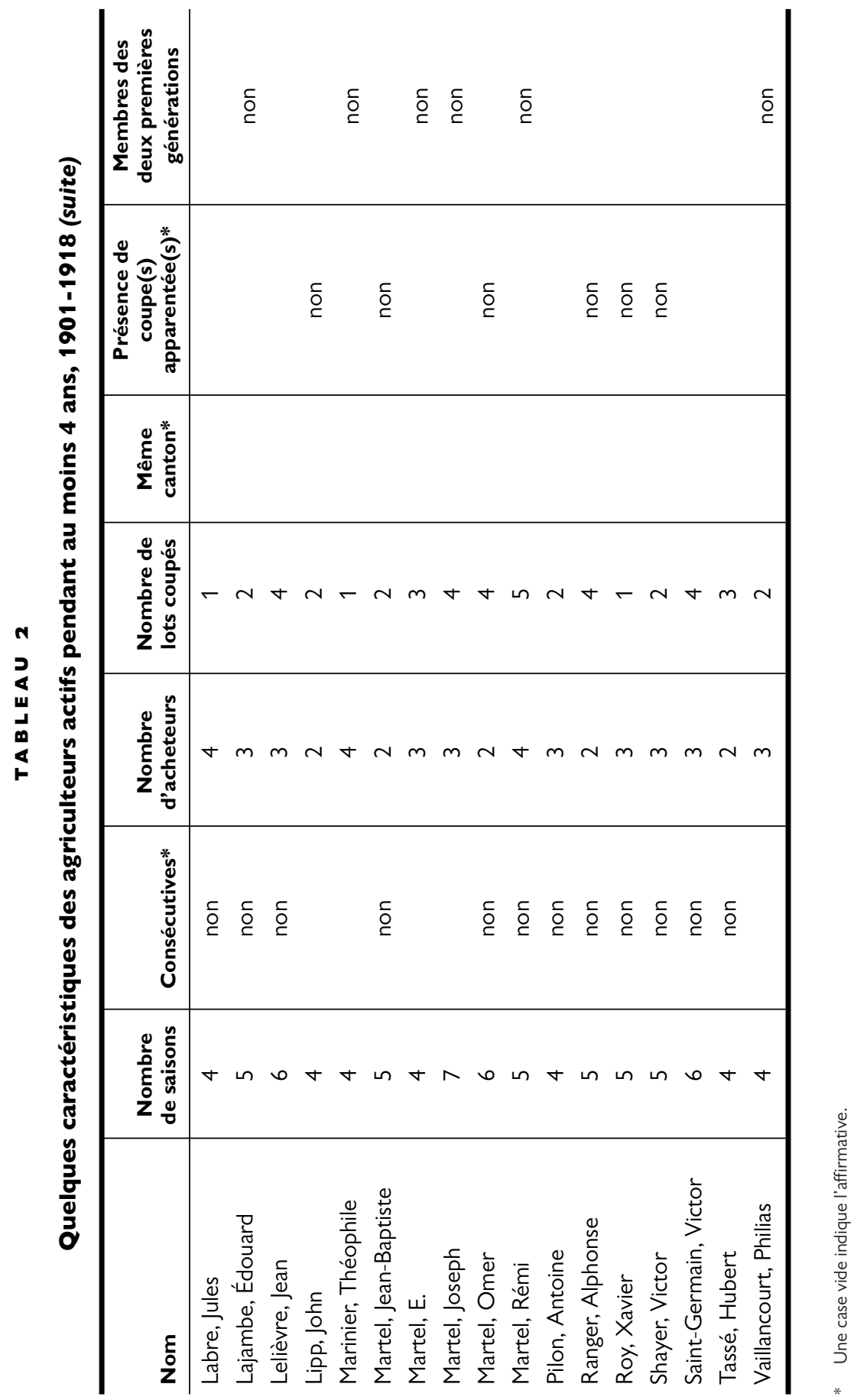


comme une seule et même entreprise. Même procédé pour la Canadian Copper Company (Inco) et la Haight \& Dickson, une société qui lui fournit du bois de chauffage. La variété des relations commerciales entretenues par les agriculteurs n'est pas sans rappeler le constat que nous faisions sur les sous-traitants en affaire avec plusieurs concessionnaires. On est loin d'un rapport de dépendance où l'agriculteur est forcé de vendre au seul acheteur de la localité. Même quand ils ne récoltent du bois que lors d'une saison, certains parviennent à vendre à deux entreprises. Selon l'évolution des prix et de la demande, l'agriculteur peut produire des traverses, du bois à pâte, quelques billes de bois de sciage et même du bois de chauffage pour les sociétés minières.

Le fait le plus surprenant dans ce tableau est que leurs récoltes proviennent presque toujours de plus d'un lot. L'agriculteur n'apparaît pas rivé sur sa terre, loin s'en faut. Il effectue sa coupe souvent sur deux et trois lots. Comment expliquer ce phénomène? L'agriculteur aurait-il déménagé? Nous ne le savons pas. En revanche, on peut mesurer jusqu’à quel point la présence d’individus apparentés expliquerait ce phénomène. Comme l'indique le tableau 2, plusieurs d'entre eux comptent au moins un parent ou un présumé parent dans les registres de coupe. Seuls les témoignages oraux et les enquêtes généalogiques pourraient établir l'ampleur du réseau de parenté de ces 36 individus, réseau qu’on soupçonne fort complexe. Faute d'information pertinente, nous avons convenu que le partage d'un même nom de famille ne permettait pas à lui seul de reconnaître un parent dans les registres et qu'il fallait aussi que le présumé parent détienne un lot à proximité. Cette méthode, qui fait abstraction des alliances de famille scellées par des mariages, implique sans doute une nette sous-estimation des liens de parenté.

La comparaison avec les agriculteurs d'une seule saison paraît significative à cet égard. Ces derniers ont beaucoup moins de parents définis selon les critères précédents. En effet, $54 \%$ des agriculteurs d'une seule saison n'ont pas de parent, contre seulement $28 \%$ des agriculteurs actifs. L'apparentement favorise l'enracinement et augmente les possibilités de vente de bois.

Cela étant admis, la cartographie de la propriété des lots demeure fort révélatrice. À titre d'illustration, nous avons retenu une partie adjacente des cantons de Hanmer et de Capreol et nous l'avons portée sur la carte 2. Cette carte trace le portrait des propriétés au 31 décembre 1912 et localise les deux tiers des individus actifs. On notera aussi que deux d'entre eux, John Lipp et Jules Labre, n'ont pas encore obtenu leur titre de propriété. 
CART E 2

\section{Les détenteurs des terres patentées d'une partie des cantons de Hanmer et de Capreol en décembre 1912}

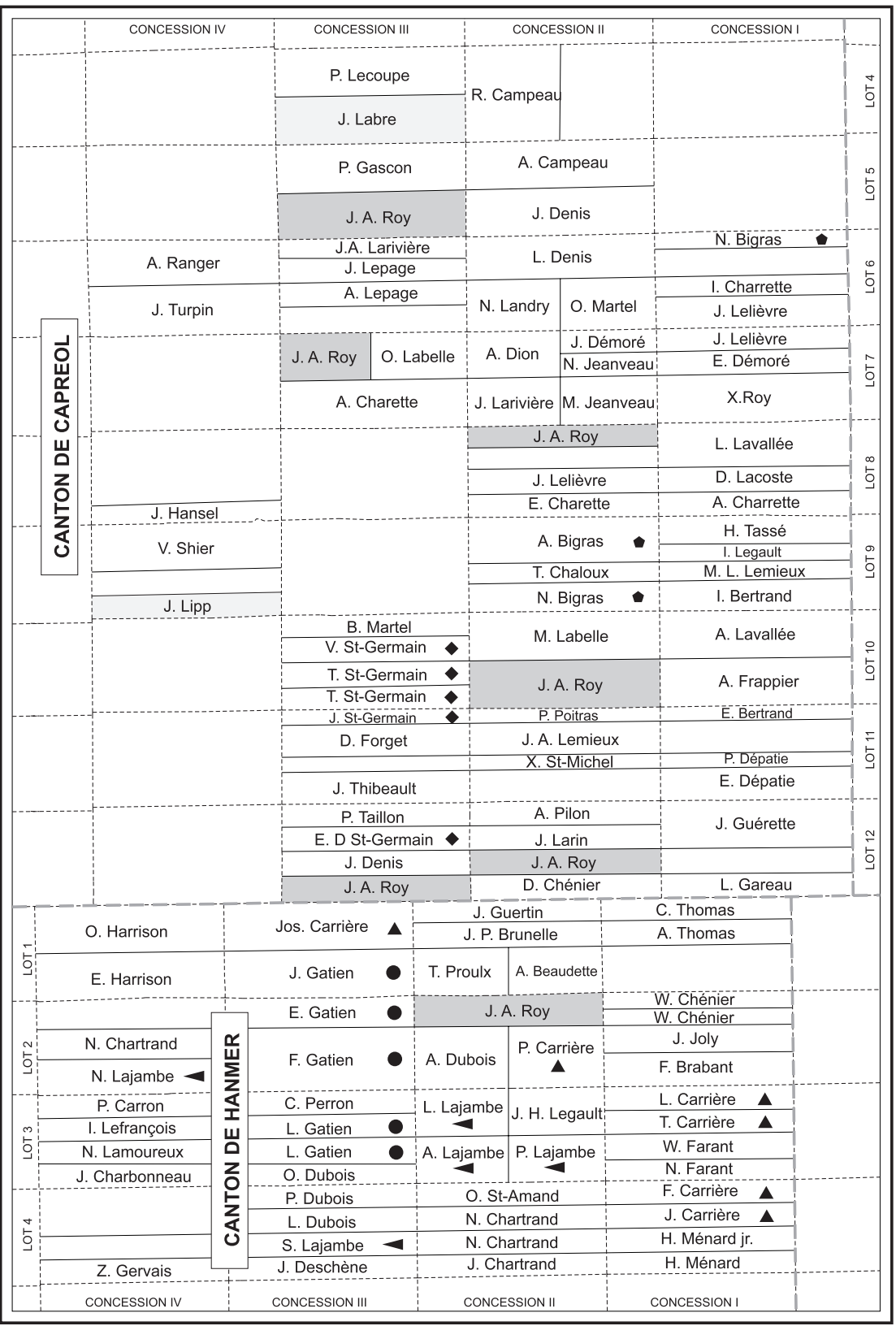

Réalisation : L.L.Larivière (Géographie, Université Laurentienne) 
Plus fondamentalement, on observe une grande proximité des agriculteurs apparentés. Les lots sont souvent voisins. Donnons quelques exemples. Les Gatien, représentés sur la carte par le symbole $\bullet$, occupent la concession III à Hanmer. Il y a aussi les Carrière (con. I, II et III, Hanmer, $\mathbf{A}$ ) et les SaintGermain (con. III, Capreol, $\bullet$ ), sans compter d'autres qu'on peut reconnaître sur la carte. Cette proximité d'agriculteurs apparentés est-elle une manifestation d'un pluriétablissement, c'est-à-dire de l'acquisition de lots additionnels en vue d'établir des fils ${ }^{46}$ ? Peut-être.

Un cas mérite d'être souligné ici, bien que ce ne soit pas celui d'un agriculteur. Il s'agit de Joseph Alphonse Roy, premier curé de la paroisse SaintJacques de Hanmer. L'étendue de ses propriétés foncières pourrait étonner ${ }^{47}$. Mais en fait, tout se passe comme s'il s'agissait d'une stratégie pour veiller à l'homogénéité — religieuse et peut-être culturelle — de sa paroisse. C'est du moins l'hypothèse que nous voudrions soumettre. Le curé rachète les terres de ceux qui partent pour les revendre vraisemblablement à de nouveaux paroissiens le temps venu. Nouveaux paroissiens qu'il se permet sans doute de sélectionner. Le curé jouerait ainsi un rôle beaucoup plus actif qu'on ne l'avait cru dans la colonisation des terres. Mais comme notre propos est d'évaluer et d'identifier les mécanismes sousjacents aux activités forestières sur les lots, nous laisserons à d'autres le soin d'examiner plus à fond les stratégies familiales et paroissiales débouchant sur la prise de terre.

Dernier élément de la coupe sur les lots agricoles : les volumes récoltés. La plupart des coupes sont à l'image de celles observées dans le tableau 1, c'est-à-dire de dimension familiale. Un père avec quelques fils peut, au cours d'un hiver, aligner une centaine de cordes de bois à pâte, ou quelques milliers de billes de bois. Mais cela ne reflète pas toute la réalité, car on rencontre régulièrement des volumes qui dépassent le niveau de production d'une famille.

Un peu arbitrairement, nous avons défini un seuil maximal qu'une famille paysanne ne pouvait pas dépasser, soit l'équivalent de 200 cordes de bois ou 100000 pmp. Au total, 91 coupes ont été examinées, ainsi que les 66 individus concernés. Le portrait brossé est similaire à celui des agriculteurs actifs pendant plusieurs saisons : forte présence des membres des

46. Voir G. Bouchard, Quelques arpents..., op. cit., voir notamment 212-220.

47. Précisons que le clergé séculier ne fait pas vœu de pauvreté. Comme nous l'indiquait notre collègue Gaétan Gervais, il faudrait sans doute que les historiens s'intéressent un jour aux affaires financières du clergé séculier. 
premières générations (64\%) ou d'éventuels parents(15\%). Près du quart d'entre eux enregistrent un double contrat au moins une saison et quelques-uns seulement maintiennent ces niveaux de coupe durant trois saisons. Encore ici, la dimension éphémère de ces activités ressort nettement.

Quelques coupes se rapprochent de celles des chantiers forestiers, avec plus de 1000 cordes. Joseph Brunet, présenté au tableau 1, n’est pas un cas unique. Quelques véritables chantiers ont été vraisemblablement mis en place sur les lots, sans compter d'autres formes d'organisation de coupe pour les volumes moins importants. Et se dégage de tout cela l'image d'une collaboration entre familles. Collaboration facilitée par le voisinage et les rapports de parenté. Un hiver, deux familles ou plus feront chantier sur tel lot, afin de profiter d'un marché favorable. La famille n'est pas contrainte à ses seuls membres immédiats pour faire chantier et cela doit être retenu.

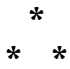

En réunissant les différents éléments présentés, il se dégage une chronologie des activités forestières en trois temps relativement brefs. D’ailleurs, cette durée a été probablement raccourcie par la présence immédiate d'agriculteurs qui colonisent la région et qui forcent en quelque sorte les entreprises forestières à récolter rapidement la matière ligneuse. Autrement dit, les mécanismes à l'œuvre ont été condensés dans le temps et ont pu même être amplifiés par le passage du chemin de fer transcontinental.

Le premier temps : celui des grands pins servant au sciage. Au cours des deux premières saisons, on récolte, par exemple, plus d'un million de billots dans quatre cantons. Peu de bois à pâte y est récolté. Des entreprises, dont les scieries, sont sises à l'extérieur de la région et accaparent des concessions forestières. Les récoltes prennent de l'ampleur, principalement dans de gros chantiers forestiers gérés par des contremaîtres. La main-d'œuvre embauchée provient surtout de l'extérieur de la région et n'est pas homogène sur le plan ethnique.

Au cours de l'étape suivante, aucun produit forestier ne domine. Les sous-traitants, sans doute déjà présents dès le début en raison de l'urgence d'abattre les arbres avant l'installation des colons, sont plus nombreux, de sorte que les chantiers affichent des dimensions plus modestes. Parmi ces sous-traitants, un certain nombre, cette fois, provient de la région immédiate, donnant à penser que la main-d'œuvre est partiellement locale. Les 
agriculteurs ont aussi commencé à vendre des produits forestiers, comme le bois à pâte; ce qui explique qu'il y a eu parallèlement une bonne récolte de bois divers - destinée surtout aux traverses de chemin de fer.

La dernière étape se caractérise d'abord par l'arrivée de concessionnaires et d'intermédiaires locaux, mais aussi par la disparition du bois de sciage. Les concessions forestières en déclin sont rachetées et des marchands de bois sillonnent la campagne à l'affût de matière ligneuse de seconde qualité et de leur mise en vente par les agriculteurs. Plus nombreux, ces derniers font même chantier parfois sur les lots en réunissant quelques familles voisines et (ou) apparentées. Parmi les agriculteurs les plus actifs, ceux qui font partie des familles pionnières comptent sur un réseau de parents. Même si généralement la coupe déclarée est celle d'une famille, il arrive occasionnellement qu'elle exige le travail de membres extérieurs à la famille immédiate.

Il ne faudrait pas croire cependant que les récoltes forestières sur les lots occupent une place centrale dans les activités agricoles. Le contraire est plutôt vrai, car la moitié des premiers détenteurs de lots n'ont pas vendu de bois; et, parmi ceux qui déclarent une récolte, plus de la moitié ne le font que pendant une seule saison. La vente de bois reste une activité accessoire. Une fois les concessionnaires assouvis et le bois des bâtiments et du chauffage mis de côté, les récoltes sont nécessairement maigres. Reste le travail dans les chantiers, dont il est difficile de mesurer l'importance, et qui compose un autre volet de cette pluriactivité. 